\title{
Tratamento eletroquímico de azo corante Acid Red 27 em solução aquosa usando anodo de $\mathrm{Ti} / \mathrm{Ru}_{0,3} \mathrm{Ti}_{0,4} \mathrm{Sn}_{0,3} \mathrm{O}_{2}$ Electrochemical treatment of Acid Red 27 azo dye in aqueous solution using $\mathrm{Ti} / \mathrm{Ru}_{0,3} \mathrm{Ti}_{0,4} \mathrm{Sn}_{0,3} \mathrm{O}_{2}$ anode
}

\author{
Maria Lucimar Maranhão Lima | Ana Carine de Sousa Monte | Bárbara Costa Albuquerque \\ Maria lonete Chaves Nogueira | Marisete Dantas de Aquino | Rinaldo dos Santos Araújo
}

Data de entrada: 14/11/2013 | Data de aprovação: 25/11/2013

DOI: http://dx.doi.org/10.4322/dae.2014.120

Resumo

Neste trabalho foi avaliada a eficiência de processos eletro-oxidativos na degradação à temperatura ambiente do azo corante Acid Red 27 em meio aquoso em diferentes concentrações (5, 10, 15 e 25 $\mathrm{mg} / \mathrm{L})$ e sob diferentes potenciais de eletrólise $(1,0 ; 1,25 ; 1,5$ e 2,5V) usando como fase eletroquímica ativa óxidos de $\mathrm{TiO}_{2}, \mathrm{RuO}_{2}$ e $\mathrm{SnO}_{2}$ sobre suporte de titânio metálico. Para a degradação eletroquímica foi utilizado o anodo dimensionalmente estável de Ti/ $\mathrm{Ru}_{0,3} \mathrm{Ti}_{0.4} \mathrm{Sn}_{0,3} \mathrm{O} 2$ preparado por decomposição térmica e caracterizado a partir das técnicas de microscopia eletrônica de varredura e energia dispersiva de raio X. Os resultados obtidos mostraram degradações superiores a 88\% em todas as concentrações investigadas para o potencial de $2,5 \mathrm{~V}$. $\mathrm{O}$ comportamento cinético encontrado foi tipicamente de primeira ordem.

Palavras-chave: Tratamento eletroquímico, Acid Red 27, Ti/ $\mathrm{Ru}_{0.3} \mathrm{Ti}_{0.4} \mathrm{Sn}_{0,3} \mathrm{O}_{2}$, Degradação, Cinética.

\section{Abstract}

In this work was investigated the efficiency of the electrooxidation processes in the degradation of azo dye Acid Red 27 at room temperature in aqueous solution at different concentrations (5, 10, 15 and 25 $\mathrm{mg} / \mathrm{L})$ and under different potential electrolysis (1.0, 1.25, 1.5 and 2.0V) using as active electrochemical phase oxides type $\mathrm{TiO}_{2}, \mathrm{RuO}_{2}$ and $\mathrm{SnO}_{2}$ supported on titanium. For the electrochemical degradation was used dimensionally stable anode of $\mathrm{Ti} / \mathrm{Ru}_{0,3} \mathrm{Ti}_{0,4} \mathrm{Sn}_{0,3} \mathrm{O}_{2}$ prepared by thermal decomposition and characterized from the techniques of scanning electron microscopy and energy dispersive $X$-ray .The results showed high degradtion (> 88\%) for all the concentrations investigated under potential of 2.0V. The kinetic behavior was typically of first order.

Key-words: Electrochemical treatment, Acid Red 27, Ti/Ru ${ }_{0,3} \mathrm{Ti}_{0,4} \mathrm{Sn}_{0,3} \mathrm{O}_{2}$, Degradation, Kinetics. 


\section{Introdução}

As evidencias da degradação ambiental estão cada vez mais claras. Nesse contexto, o grande desafio é manter a capacidade suporte dos ecossistemas e ampliar a compreensão do ambiente e das novas tecnologias no que diz respeito à preservação da qualidade ambiental. A qualidade dos recursos hídricos está entre as principais preocupações ambientais, considerando que tanto a qualidade quanto a quantidade de água disponível sempre foi um indicador importante na determinação do bem-estar da sociedade.

As novas tecnologias aplicadas de modo adequado vêm ganhando espaço no desenvolvimento de processos de controle e monitoramento da poluição como um todo e, em particular na poluição da água e das águas residuárias. Sabe-se que substâncias tóxicas têm forte influencia nos ecossistemas aquáticos e nos organismos que nele vivem, podendo entrar na água por aporte direto ou escoamento superficial, onde a mobilidade das substâncias poluidoras dependerá da fluidez do meio. Considerando que os contaminantes na água deslocam-se com as correntes por processos diversos, tais como: mistura, difusão, sedimentação, suspensão de partículas entre outros, faz-se necessário a busca constante de tecnologias de tratamento objetivando diminuir os possíveis danos ambientais.

Os efluentes de um modo geral e, em particular, os provenientes da indústria têxtil, devido sua complexidade, possuem grande probabilidade de causar problemas ambientais por conter substâncias persistentes, tóxicas e apresentar tendência à bioaculmulação, vem ganhando atenção especial no que diz respeito ao desenvolvimento de processos de tratamento para minimizar os impactos adversos ao ambiente. A questão desafiadora consiste na escolha e utilização de técnicas adequadas para degradação de substâncias potencialmente poluidoras presentes nas águas residuárias em especial nos efluentes oriundos da indústria têxtil, considerando que entre outros compostos, possuem grande quantidade de corantes e, do ponto de vista ambiental, os corantes contribuem para a geração de efluentes significativamente tóxicos, com elevada carga de compostos orgânicos recalcitrantes e, em muitos casos são descartados no meio ambiente de forma inadequada.

De acordo com a literatura aproximadamente $15 \%$ da produção mundial de corantes é descar- tado ao meio ambiente durante a síntese, processamento ou aplicação (DOS SANTOS, et al., 2011). Isso do ponto de vista ambiental é um grande problema, considerando que esse valor representa o lançamento de cerca de 1,5 ton/dia de efluente com corante para o meio ambiente. Nesses efluentes, os corantes azo são predominantes, por pertencerem à maior classe de corantes, com ampla variedade de cores (ABRAHAM et al., 2003). Os corantes do tipo azo possuem importância significativa, englobando cerca de 50\% dos corantes comerciais, além de serem mais estudados que os de outras classes.

Frente a isso, cada vez mais esses compostos merecem atenção especial por apresentarem um significativo potencial poluidor, devido à possibilidade de decomposição natural produzindo outras espécies também prejudiciais aos corpos hídricos e devido à quantidade significativa que é lançada anualmente ao meio ambiente.

Dentre os vários tipos de moléculas empregadas destacam-se os corantes reativos, os quais são bastante usados para tingir celulose e algodão. Os corantes reativos apresentam uma complexa estrutura química. A presença da estrutura do grupamento azo $-\mathrm{N}=\mathrm{N}$ - é responsável pela cor do corante, sendo necessário o rompimento dessa ligação para ocorrer a descoloração. O potencial de impacto dessas substâncias está relacionado ao fato de conter substâncias não biodegradáveis e potencialmente perigosas.

$\mathrm{Na}$ literatura diversos estudos indicam que compostos orgânicos de interesse ambiental podem ser eletro-oxidados eficientemente em soluções aquosas, utilizando-se eletrodos óxidos mistos contendo Ti, Ru e Sn (PANIZZA et al., 2006; CHELLAMMAL et al., 2012).

Segundo Martínez-Huiltlee e Brillas (2009) os processos eletroquímicos por meio de reações de oxi-redução possibilitam o tratamento de substâncias tóxicas utilizando mecanismos mais facilmente controlados com pequenas quantidades de agentes oxidantes. Panizza et al. (2000), Torres et al. (2003), Kariyajjanavara et al. (2011), Parsa et al. (2013) consideram como vantagem, a versatilidade do tratamento eletroquímico, bem como a maior eficiência energética, compatibilidade ambiental e o baixo custo de operação. No tratamento eletroquímico a degradação é realizada através do potencial ou corrente aplicada que geram eletroliticamente reagentes secundários com elevado poder oxidante (SONI et al., 
2011, SOLIS et al., 2012), não requerendo a utilização quantitativa de outros insumos químicos.

Vários pesquisadores investigaram a preparação, caracterização e aplicação de eletrodos dimensionalmente estáveis (ADE) entre as décadas de 1970 e 1980. Nos dias atuais os ADEs ainda são preparados utilizando-se as técnicas de decomposição térmica da mistura dos sais precursores sobre o suporte metálico rugoso.

Em geral, os processos eletroquímicos utilizados no tratamento de poluentes ambientais tem como base a reação direta destes compostos na superfície dos eletrodos ou na reação indireta envolvendo a formação de espécies eletroativas no eletrodo e que reage com as substâncias poluentes. (RAJESHWAR e IBBANEZ, 1996; FÓTI et al., 1997; ARNOLD, 2010; SONI et al., 2011).

Estudos mostram que são investigadas variações quanto ao método de preparação dos eletrodos de óxidos comerciais e quanto ao suporte metálico utilizado objetivando aumentar a resistência e a estabilidade desses eletrodos (TEREZO e PEREIRA, 2002; FORTI et al., 2003). Alguns pesquisadores já demostraram que eletrodos de óxidos podem ser também empregados em capacitores eletroquímicos para estocagem de energia, em medidores de $\mathrm{pH}$, em função da resposta dos óxidos frente às mudanças de $\mathrm{pH}$ e na degradação eletroquímica de compostos orgânicos (KIM e KIM, 2001; TRASATTI, 1995; KINOSHITA et al., 1986).

De acordo com a literatura reação de oxidação eletroquímica ocorre inicialmente com a formação de radicais hidroxilos $\left(\mathrm{MO}_{\mathrm{x}}\left({ }^{\circ} \mathrm{OH}\right)\right)$ originados da descarga eletroquímica da água adsorvida fisicamente na superfície do eletrodo (COMNINELLIS, 1994). O mecanismo para a degradação eletrocatalítica de compostos orgânicos utilizando anodo dimensionalmente estável (ADEs) envolve as seguintes reações:

$$
\begin{array}{r}
\mathrm{MO}_{\mathrm{X}}+\mathrm{H}_{2} \mathrm{O} \rightarrow \mathrm{MO}_{\mathrm{X}}(. \mathrm{OH})+\mathrm{H}^{+}+\mathrm{e}^{-} \\
\text {Equação (1) } \\
\mathrm{R}+\mathrm{MO}_{\mathrm{X}}(. \mathrm{OH}) \rightarrow \mathrm{MO}_{\mathrm{X}}+\mathrm{CO}_{2}+\mathrm{H}^{+}+\mathrm{e}^{-} \\
\text {Equação (2) } \\
\mathrm{MO}_{\mathrm{X}}(. \mathrm{OH}) \rightarrow \mathrm{MO}_{\mathrm{X}}+1 / 2 \mathrm{O}_{2}+\mathrm{H}^{+}+\mathrm{e}^{-} \\
\text {Equação (3) }
\end{array}
$$

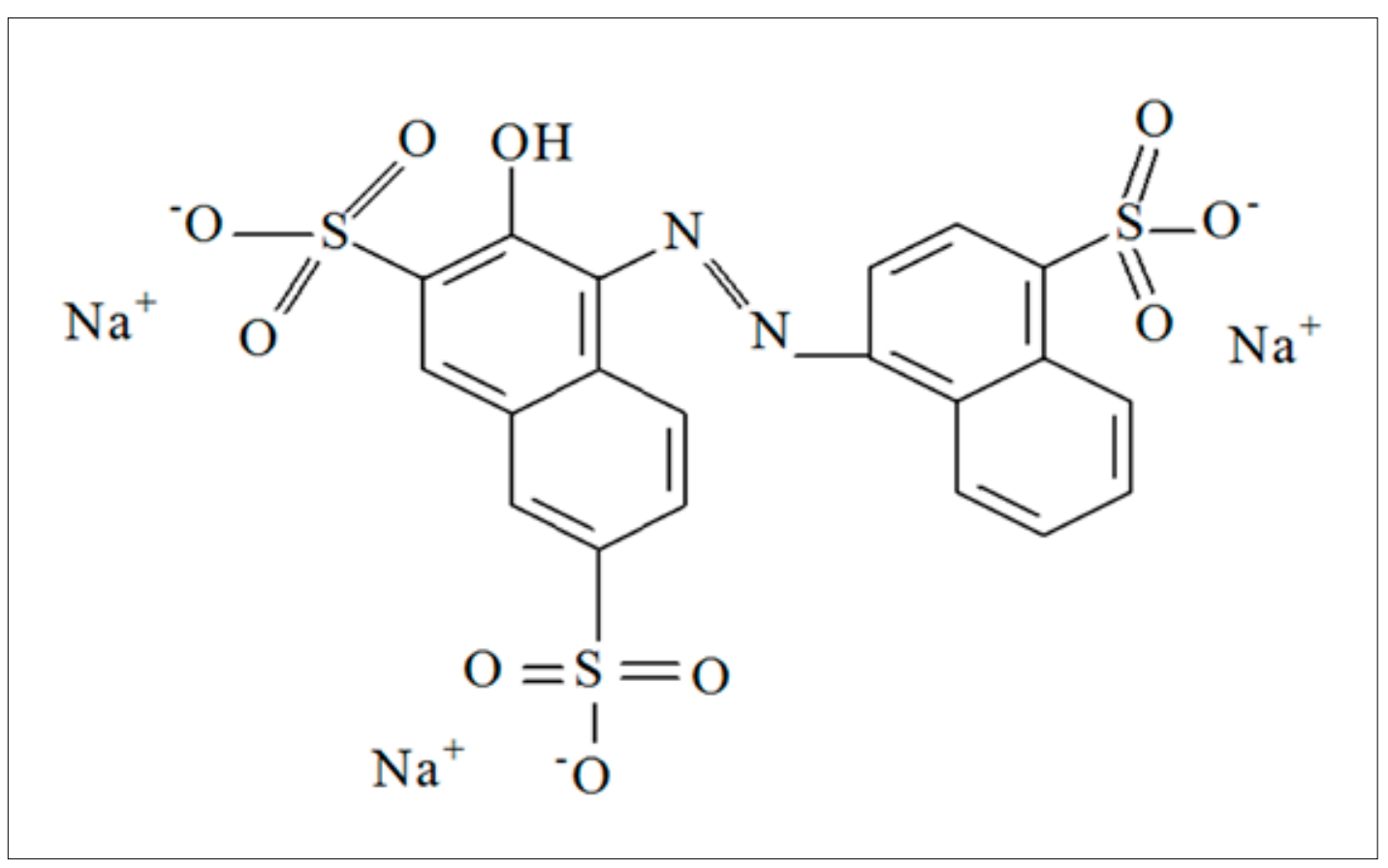

Figura 1 - Estrutura Molecular do azo corante Acid Red 27 (AR 27). 
Onde: $\mathrm{MO}_{\mathrm{x}}$ corresponde ao sítio óxido metálico em seu estado normal, $\mathrm{MO}_{\mathrm{x}}(\cdot \mathrm{OH})$ representa o sítio oxido metálico com radical hidroxilo adsorvido e $\mathrm{R}$ representa o composto orgânico.

Assim neste estudo foi avaliada a eficiência de remoção de cor e degradação do corante AR 27 (Figura 1) em meio aquoso e à temperatura ambiente usando processo eletroquímico com anodo de $\mathrm{Ti} / \mathrm{Ru}_{0,3} \mathrm{Ti}_{0,4} \mathrm{Sn}_{0,3} \mathrm{O}_{2}$. O corante Acid Red 27 devido a sua intensa coloração encontra aplicação variada em segmentos da indústria têxtil, de cosmético, farmacêutica e alimentícia. Estruturalmente possui um grupo azo $-\mathrm{N}=\mathrm{N}$ - ligado a dois anéis naftalênicos sulfonados (Figura 1) e vem sendo estudado na literatura devido a seus possíveis efeitos tóxicos e carcinogênicos (PAN et al., 2005).

\section{METODOLOGIA}

Preparação do anodo de $\mathrm{Ti} / \mathrm{Ru}_{0,3} \mathrm{Ti}_{0,4} \mathrm{Sn}_{0,3} \mathrm{O}_{2} \mathrm{e}$ montagem do sistema eletroquímico

Os eletrodos foram preparados a partir de reagentes de grau analítico $\left(\mathrm{RuCl}_{3} \mathrm{SnCl}_{2} \cdot 2 \mathrm{H}_{2} \mathrm{O}\right.$ e, $\mathrm{TiCl}_{3} 15 \%$ em $\mathrm{HCl}$ ) fornecidos pela Sigma-Aldrich e água ultrapura obtida em sistema UHQ OS-MK3 ELGA. As soluções foram preparadas separadamente na concentração de 1 mol.dm $^{3}$ partindo da dissolução dos respectivos cloretos em álcool isopropílico. A solução precursora foi preparada com a mistura das soluções de $\mathrm{RuCl}_{3}$ $\mathrm{SnCl}_{2} \cdot 2 \mathrm{H}_{2} \mathrm{O}$ e $\mathrm{TiCl}_{3}$ na proporção molar de 3:4:3, respectivamente.

Para a preparação dos eletrodos utilizou-se um substrato de titânio proveniente da TiBrasil Ltda de alta pureza com área de $1 \mathrm{~cm}^{2}$ e $0,25 \mathrm{~mm}$ de espessura. Inicialmente os substratos foram mecanicamente polidos com lixa d'água de granulometria 120, objetivando melhorar a aderência do suporte para o posterior recobrimento com os óxidos desejados. Em seguida realizaram-se os procedimentos de limpeza por enxágue com água destilada para eliminação de impurezas seguido de desengraxe por imersão da placa de titânio em $\mathrm{NaOH}$ 10\% por cerca de 10 minutos. A eliminação do excesso de álcali foi por meio de lavagem com água destilada. O substrato de titânio foi então submetido a ataque químico por 10 minutos em $\mathrm{H}_{2} \mathrm{C}_{2} \mathrm{O}_{4} 10 \%$ a quente com a finalidade de aumentar a rugosidade e facilitar a adesão da solução precursora à superfície do substrato. Os suportes de titânio foram então secos com jato de ar quente e pesados para dar início ao recobrimento com os óxidos.

A massa de óxido a ser depositada sobre o titânio foi determinada utilizando a equação 4 a seguir:

$$
\begin{array}{r}
\mathrm{m}(\mathrm{g})=\mathrm{A} . \varnothing\left[\mathrm{xRuO}_{2 . \rho} \mathrm{RuO}_{2}+\mathrm{xTiO}_{2 . \rho} \mathrm{TiO}_{2}+\mathrm{xSnO}_{2 . \rho} \mathrm{SnO}_{2}\right] \\
\text { Equação (4) }
\end{array}
$$

Onde: $A$ = área do suporte metálico,

$\varnothing=$ espessura nominal do filme de óxido a ser depositado,

$x=$ fração molar,

e $\rho=$ densidade do respectivo óxido.

O recobrimento das placas de titânio pelos óxidos foi realizado por meio da tradicional técnica

Fio de Cobre

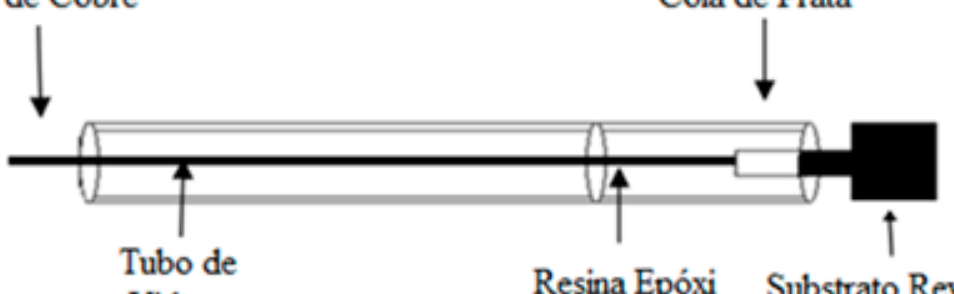

Vidro

com camada de óxido

Figura 2 - Diagrama esquemático da montagem do eletrodo de trabalho empregado nos estudos de eletro-oxidação. Adaptado de Nogueira (2009). 
de pincelamento (LASSALI et al., 1994 e ALVES et al., 1994), que consiste na pintura repetida, em ambos os lados das placas, com a mistura das soluções precursoras recém-preparadas.

Após pintura os substratos foram secos com jato de ar quente e levados ao forno mufla por 5 minutos a temperatura de $500^{\circ} \mathrm{C}$. Após termo decomposição dos cloretos inorgânicos os eletrodos foram acondicionados em um dessecador até atingirem a temperatura ambiente para então serem pesados. Este procedimento foi repetido até obtenção de uma massa de aproximadamente $2 \mathrm{mg}$ de depósito, correspondente a uma camada de óxido com espessura de $2 \mu \mathrm{m}$. A etapa de calcinação a $500^{\circ} \mathrm{C}$ foi mantida por um período de tempo de $1 \mathrm{~h}$ para estabilização dos óxidos formados e eliminação de possíveis impurezas residuais. Após a deposição da camada de óxido, realizou-se a montagem final do eletrodo esquematizado de acordo com a Figura 2.

Os procedimentos eletroquímicos foram realizados em uma célula de vidro com volume reacional de $60 \mathrm{~mL}$ composta por um único compartimento e uma tampa de teflon com quatro orifícios sendo três destinados a fixação dos eletrodos: eletrodo de trabalho $\left(\mathrm{Ti} / \mathrm{Ru}_{0,3} \mathrm{Ti}_{0.4} \mathrm{Sn}_{0,3} \mathrm{O}_{2}\right)$, eletrodo auxiliar $(\mathrm{Pt})$ e eletrodo de calomelano saturado $\left(\mathrm{Hg} / \mathrm{Hg}_{2} \mathrm{Cl}_{2} \cdot \mathrm{KCl}\right)$ usado como eletrodo de referência. $\mathrm{O}$ quarto orifício é destinado à saída de gases gerados durante o processo de degradação eletrocatalítica.

\section{Caracterização física e química dos eletrodos}

A caracterização da estrutura física da superfície dos eletrodos foi realizada por meio da técnica de Microscopia Eletrônica de Varredura (MEV) em um microscópio PHILIPS, modelo XL30. A análise da composição química do óxido depositado foi obtida por meio da técnica semi-quantitativa de energia dispersiva de raios- $X$ (EDX) utilizando um sistema LINK Analytical, modelo QX-2000 acoplado ao microscópio eletrônico PHILIPS.

\section{Caracterização eletroquímica dos eletrodos e en- saios de eletro-oxidação}

A caracterização eletroquímica do eletrodo de $\mathrm{Ti} / \mathrm{Ru}_{0,3} \mathrm{Ti}_{0.4} \mathrm{TiSn}_{0,3} \mathrm{O}_{2}$ e a estabilidade da camada de óxido foi verificada por meio da técnica de voltametria cíclica à temperatura de $28^{\circ} \mathrm{C}$ utilizando um potenciostato/galvanostato AUTOLAB modelo PGSTAT 101 conectado a um computa- dor e controlado pelo software NOVA 1.5, possibilitando a aquisição e tratamento dos dados experimentais obtidos.

Experimentalmente para os estudos de degradação eletrocatalítica utilizaram-se soluções aquosas sintéticas do corante Acid Red 27 (AR 27) em diferentes concentrações e uma solução de $\mathrm{Na}_{2} \mathrm{SO}_{4}$ 0,25mol/L como eletrólito suporte. Os ensaios foram conduzidos à temperatura ambiente $\left(28^{\circ} \mathrm{C}\right)$ em $\mathrm{pH}$ natural $(6,0)$ e sob potenciais de $1,0 \mathrm{~V} ; 1,25 \mathrm{~V} ; 1,5 \mathrm{~V}$ e $2,5 \mathrm{~V}$. As alíquotas para o acompanhamento cinético da degradação foram retiradas em intervalos regulares de 20 , 40, 60, 80, 100, 120, 150, 180 e 240 minutos.

As análises da concentração residual de corante foram realizadas por espectroscopia de absorção molecular utilizando-se um espectro Thermo Scientific modelo Genesys 10UV de feixe simples a 520nm, comprimento de onda de máxima absorção do corante AR 27.

Os parâmetros cinéticos de descoloração/degradação relacionados ao efeito da concentração inicial do corante foram obtidos partindo-se do modelo clássico de primeira ordem, conforme a Equação 5 a seguir:

$$
-\frac{d c}{d t}=k_{1} C
$$

Equação (5)

\section{Onde:}

$C$ é a concentração do corante em um dado tempo reacional t;

e $k_{1}$ é a constante cinética de primeira ordem $\left(\mathrm{min}^{-1}\right)$.

$\mathrm{O}$ valor de $\mathrm{k}_{1}$ pode ser obtido de forma direta de acordo com a Equação 6, após linearização da Equação 5.

$$
\ln \frac{C_{1}}{C_{0}}=-k_{1} \cdot t \underbrace{}_{\text {Equação (6) }}
$$

Para a estimativa das eficiências de descoloração/degradação da solução do azo corante em estudo foi utilizada a Equação 7: 


$$
\text { Eficiência }(\%)=\left(1-\frac{C_{f}}{C_{0}}\right) \times 100 \%
$$

Onde:

$C_{f}$ corresponde à concentração da solução de corante a 520nm em um dado tempo reacional $t$ após o tratamento de eletro-oxidação; e $C_{0}$ a concentração inicial da solução do corante.

\section{Resultados e discussão}

Caracterização superficial do eletrodo de $\mathrm{Ti} / \mathrm{Ru}_{0,3} \mathrm{Ti}_{0,4} \mathrm{Sn}_{0,3} \mathrm{O}_{2}$

As análises do filme de óxido depositado no eletrodo $\mathrm{Ti} / \mathrm{Ru}_{0,3} \mathrm{Ti}_{0,4} \mathrm{Sn}_{0,3} \mathrm{O}_{2}$ por MEV mostraram uma morfologia do tipo barro rachado (cracked-mud), conforme a Figura 3.

A Figura 3A mostra uma intensa ocorrência de microfissuras na superfície eletródica, bem como deposição de material particulado, enquanto que na Figura 3B percebe-se uma nítida deposição de crosta sobre a superfície do eletrodo, com a presença de rachaduras micrométricas. Segundo a literatura esta morfologia caracterizada por fendas, fissuras e micro rachaduras confere ao
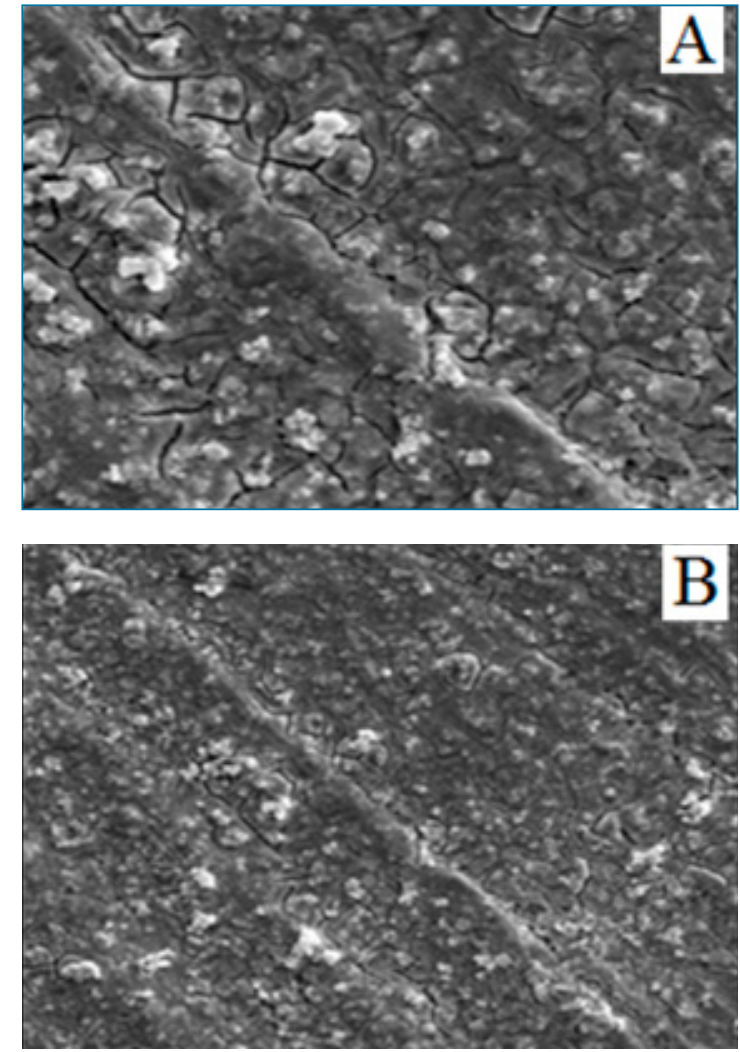

Figura 3 - Micrografias eletrônicas da superfície do anodo de $\mathrm{Ti} / \mathrm{Ru} \mathrm{o}_{0,3} \mathrm{Ti}_{0,4} \mathrm{Sn}_{0,3} \mathrm{O}_{2}$. A) aumento de $\left.1000 \mathrm{x}, \mathrm{B}\right)$ aumento de $2000 x$

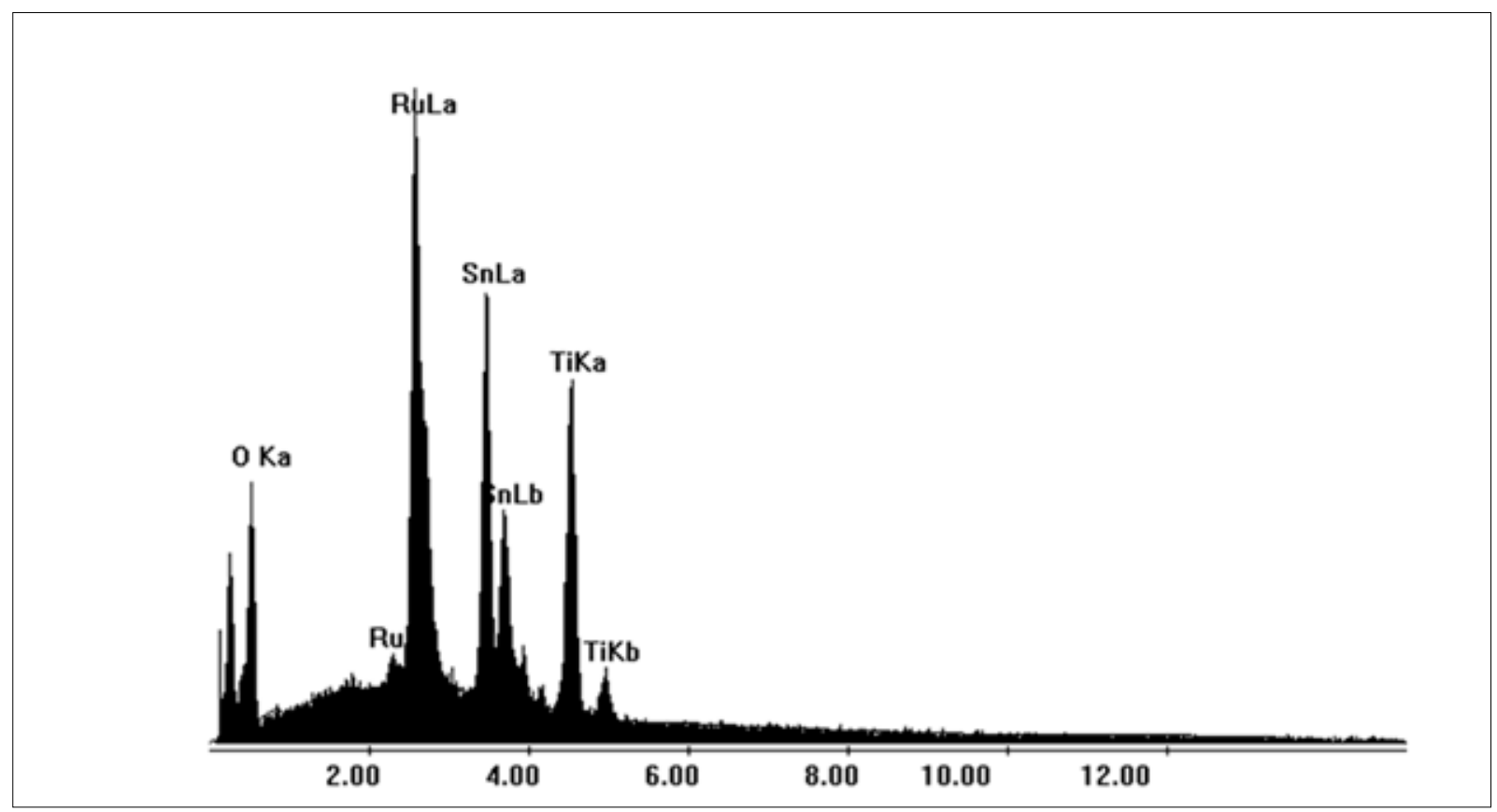

Figura 4 - Espectro EDX da superfície do anodo de $\mathrm{Ti} / \mathrm{Ru} u_{0,3} \mathrm{Ti}_{0,4} \mathrm{Sn}_{0,3} \mathrm{O}_{2}$. 
eletrodo uma elevada área superficial, o que favorece os fenômenos eletroquímicos superficiais (LIPP e PLETCHER, 1997; LANZA e BERTAZZOLI, A análise da composição percentual dos óxidos por EDX (Figura 4) mostrou valores de: 31\% de $\mathrm{RuO}_{2}, 38 \%$ de $\mathrm{TiO}_{2}$ e $31 \% \mathrm{SnO}_{2}$, os quais são muito próximos da composição nominal na solução precursora (30\% de $\mathrm{RuO}_{2}, 40 \%$ de $\mathrm{TiO}_{2}$ e $30 \%$ $\mathrm{SnO}_{2}$ ). A proximidade entre os valores apresentados evidenciam a eficiência do processo utilizado para obtenção do eletrodo em estudo.

\section{Cinética de degradação}

A cinética de descoloração do corante Acid Red 27 à temperatura ambiente $\left(28^{\circ} \mathrm{C}\right)$ nas concentrações investigadas, segundo o modelo de primeira ordem, pode ser observada a seguir na Figura 5.
Na Tabela 1 estão apresentados os valores das eficiências de degradação (\%) e constante cinética $\left(k_{1}, \mathrm{~min}^{-1}\right)$ relacionada ao modelo de primeira ordem para a degradação da solução aquosa do AR 27 sob diferentes potenciais de eletrólise nas concentrações de 5, 10, 15 e $25 \mathrm{mg} / \mathrm{L}$; e nela se verifica que o potencial de $2,5 \mathrm{~V}$ apresenta melhor desempenho eletro-oxidativo alcançando eficiências de degradação entre 88 e 94\% para todas as concentrações investigadas. Estes valores podem ser considerados muito promissores como alternativa de tratamento ambiental de azo corantes em meio aquoso, uma vez que nesta condição o efluente apresenta ausência de coloração e baixa concentração residual de matéria orgânica, conforme recomendado pela Resolução CONAMA 430/2011 que dispõe sobre as condições e padrões de lançamento
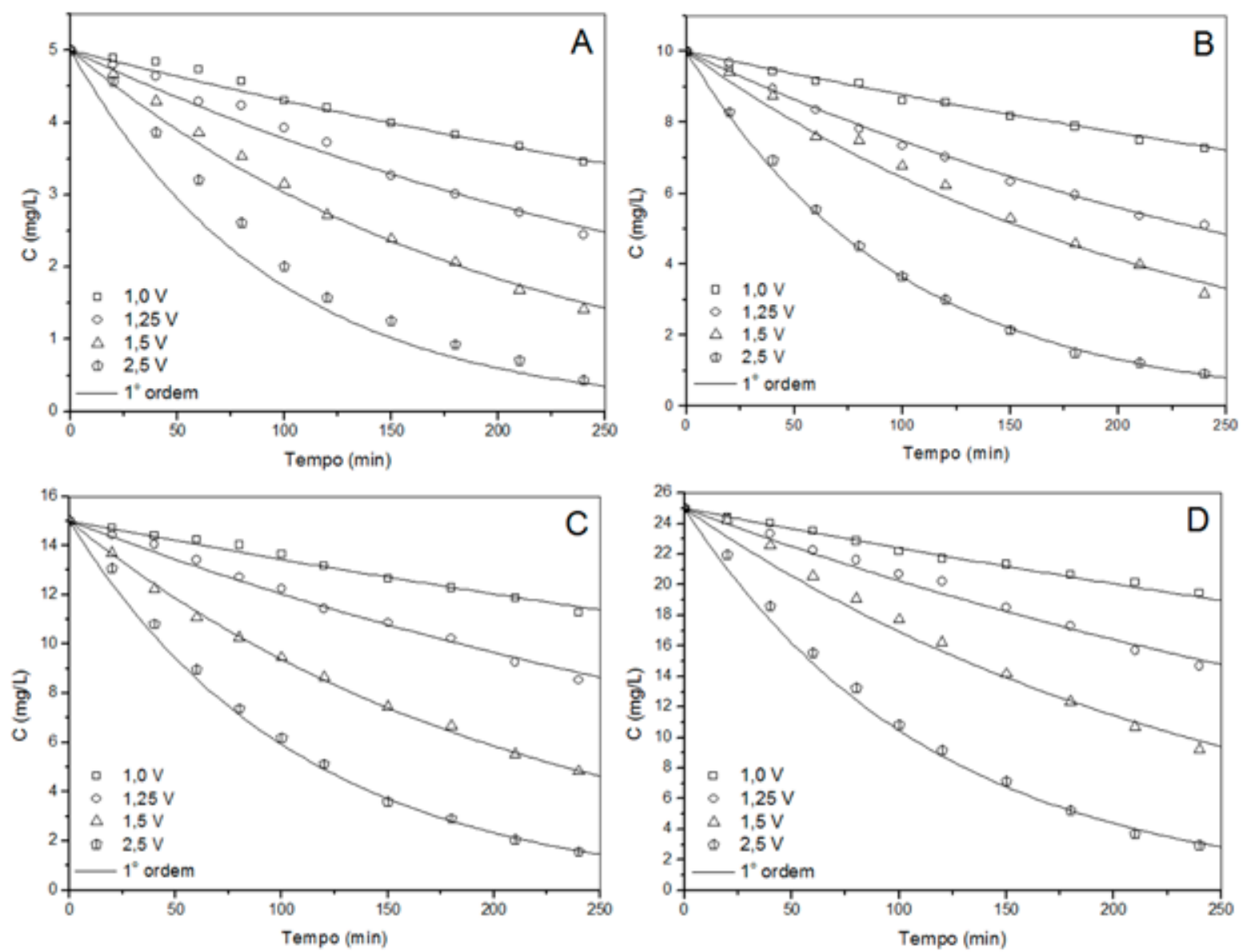

Figura 5 - Perfil cinético de primeira ordem a $28^{\circ} \mathrm{C}$ para a degradação eletroquímica do corante AR 27 nas concentrações de: $5 \mathrm{mg} / \mathrm{L}(\mathrm{A}), 10 \mathrm{mg} / \mathrm{L}(B), 15 \mathrm{mg} / \mathrm{L}(C), 25 \mathrm{mg} / \mathrm{L}(\mathrm{D})$ em diferentes potenciais utilizando o anodo de $\mathrm{Ti} / \mathrm{Ru}_{0,3} \mathrm{Ti}_{0,4} \mathrm{Sn}_{0,3} \mathrm{O}_{2 .} \mathrm{pH}=6,0$; Eletrólito: $\mathrm{Na}_{2} \mathrm{SO}_{4} 0,25 \mathrm{~mol} / \mathrm{L}$. 


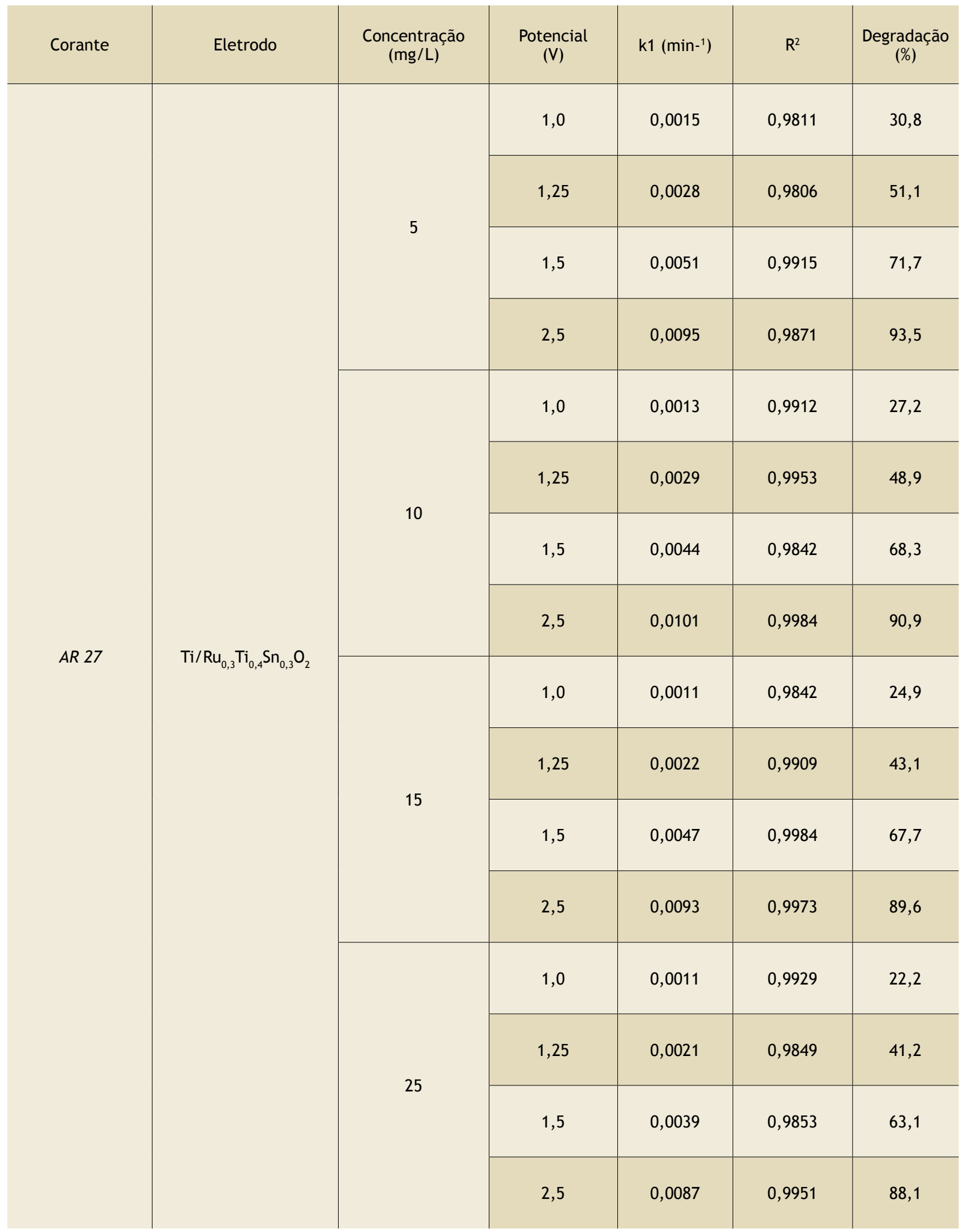

Tabela 1. Eficiências de degradação (\%) e parâmetros cinéticos a $28^{\circ} \mathrm{C}$ para a degradação eletrocatalítica do corante AR 27 sob anodo de $\mathrm{Ti} / \mathrm{Ru}_{0.3} \mathrm{Ti}_{0.4} \mathrm{Sn}_{0,3} \mathrm{O}_{2}$ em diferentes concentrações iniciais do corante e sob diferentes potenciais de eletrólise. $\mathrm{pH}=6,0$; Eletrólito: $\mathrm{Na}_{2} \mathrm{SO}_{4}$ 0,25 mol/L. 
de efluentes, em seu Art. $3^{\circ}$ determina que os efluentes de qualquer fonte poluidora somente poderão ser lançados diretamente nos corpos receptores após devido tratamento e desde que obedeçam às condições padrões de exigências dispostos nesta resolução e em outras normas aplicáveis (CONAMA 430/2011). Embora a resolução não estabeleça limite de cor para descarte de efluente, o Capítulo II, Art. $5^{\circ}, \S 2^{\circ}$ estabelece que para os parâmetros não incluídos, os padrões de qualidade a serem obedecidos são os que constam na classe na qual o corpo receptor estiver enquadrado.

Quanto aos potenciais mais baixo de 1,0 e $1,25 \mathrm{~V}$ mostraram baixa performance eletrocatalítica com percentuais de remoção de corante inferiores a $52 \%$.
Do ponto de vista cinético, em geral o modelo de primeira ordem apresenta elevados coeficientes de correlação $\left(R^{2}>0,981\right)$ justificando um mecanismo reacional controlado pela quantidade de radicais hidroxil $(\cdot \mathrm{OH})$ gerados durante a eletrólise na superfície do anodo de $\mathrm{Ti} / \mathrm{Ru}_{0,3} \mathrm{Ti}_{0,4} \mathrm{Sn}_{0,3} \mathrm{O}_{2}$, (NEPPOLIAN et al., 2002, WU et al., 2004) haja visto que para a mesma concentração inicial de corante estudada as eficiências degradativas são praticamente as mesmas apresentando uma diferença máxima de $10 \%$ de remoção entre 5 e $25 \mathrm{mg} / \mathrm{L}$.

Experimentalmente, os valores das constantes cinéticas de primeira ordem $\left(\mathrm{k}_{1}\right)$ mostram uma cinética mais favorável com a diminuição da concentração na faixa de valores investigados para o corante AR 27 devido ao efeito
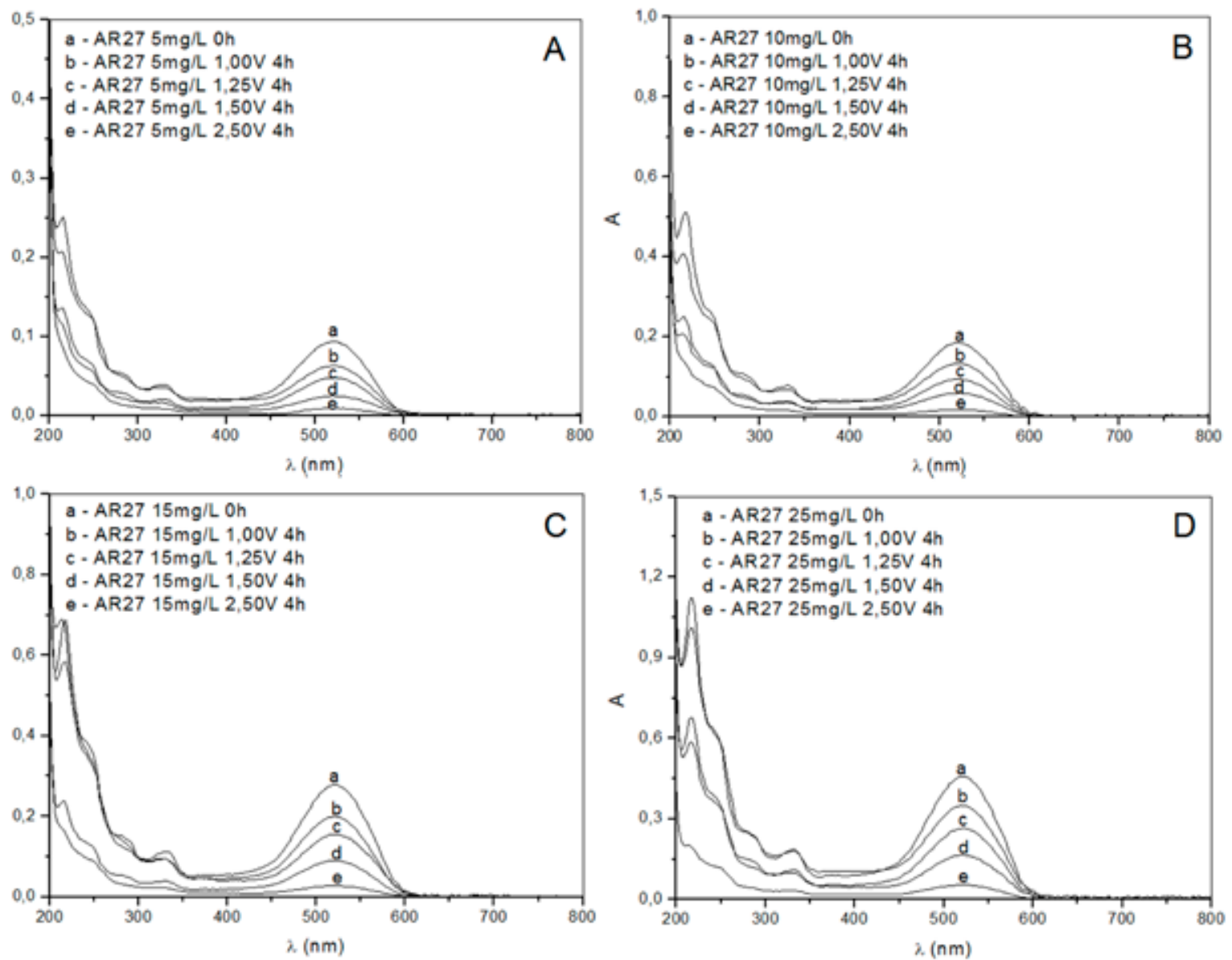

Figura 6 - Espectros de absorção molecular UV-Vis da solução residual de AR 27 após 4h de tratamento eletrooxidativo a $28^{\circ} \mathrm{C}$ sobre anodo de $\mathrm{Ti} / \mathrm{Ru}_{0,3} \mathrm{Ti}_{0,4} \mathrm{Sn}_{0,3} \mathrm{O}_{2} . \mathrm{pH}=6,0$; Eletrólito: $\mathrm{Na}_{2} \mathrm{SO}_{4}$ 0,25 $\mathrm{mol} / \mathrm{L}$. 
de absorção de menor quantidade de radicais frente ao excesso estequiométrico da molécula orgânica (NÚÑES et al., 2007). Adicionalmente a cinética é mais rápida com o aumento do potencial de eletrólise, na faixa de valores investigados o que está em conformidade com estudos anteriores de Yang et al., (2009). Resumidamente para o efeito do potencial Os valores de $\mathrm{k}_{1}\left(\mathrm{~min}^{-1}\right)$, independente da concentração aplicada, seguiram a ordem: $2,5 \mathrm{~V}>>$ $1,5 \mathrm{~V}>1,25 \mathrm{~V}>1,0 \mathrm{~V}$.

A Figura 6 apresenta os espectros de absorção molecular do azo corante AR 27 para o tratamento eletrocatalítico sob as diferentes condições de concentração e potenciais aplicados.

Na Figura 6 observa-se o decaimento das bandas de absorção molecular em 220 e $350 \mathrm{~nm}$, características dos grupos benzênicos e naftênicos, o que evidencia uma apreciável degradação/mineralização da molécula do corante. Em termos analíticos foram identificados por cromatografia de íons (resultados não apresentados) espécies como: formato, acetato, maleato, nitrato, carbonato e sulfato no efluente final pós-tratamento eletroquímico. Quanto à descoloração do corante, à medida que o potencial de eletrólise aumenta constata-se um expressivo decaimento da banda cromófora a 520nm característico do azo corante AR 27.

\section{Conclusões}

Para o azo corante estudado, o AR 27, o tratamento eletrocatalítico com anodo de $\mathrm{Ti} /$ $\mathrm{Ru}_{0,3} \mathrm{Ti}_{0,4} \mathrm{Sn}_{0,3} \mathrm{O}_{2}$ mostrou-se muito promissor atingindo eficiências de degradação acima de $88 \%$ para um potencial aplicado de $2,5 \mathrm{~V}$.

Para a descoloração/degradação do corante em estudo observa-se um aumento da eficiência oxidativa com o incremento do potencial de eletrólise na faixa de 1 a $2,5 \mathrm{~V}$, conforme verificado a partir do decaimento intenso das bandas de absorção a 280, 350 e 520nm para os ensaio de eletro-oxidação nas diferentes concentrações iniciais de corante aplicadas (5 a $25 \mathrm{mg} / \mathrm{L}$ ).

Considerando que os corantes azos quando degradados por processos convencionais geram produtos potencialmente tóxicos e carcinogênicos, técnicas de tratamento de efluentes que removam tais poluentes de modo seguro e ambientalmente adequados devem ser melhor estudados. Em geral, os bons resultados de atividade catalítica encontrados, evidenciam a técnica eletroquímica com anodo dimensionalmente estável de $\mathrm{Ti} / \mathrm{Ru}_{0,3} \mathrm{Ti}_{0,4} \mathrm{Sn}_{0,3} \mathrm{O}_{2}$ como alternativa de tecnologia interessante para o tratamento ambiental em sistemas aquosos contendo contaminantes tipo azo corantes.

\section{Referências}

ALVES, V. A.; SILVA, L. A.; BOODTS, J. F. C.; TRASATTI, S. Kinetics and mechanism of oxygen evolution on $\mathrm{IrO}_{2}$-based electrodes containing $\mathrm{Ti}$ and $\mathrm{Ce}$ acidic solutions. Electrochimica Acta, v. 39, n. 11-12, p. 1585-1589, 1994.

ABRAHAM, T. E., SENAN, R. C., SHAFFIQU, T. $S$. Bioremediation of textile azo dyes by an aerobic bacterial consortium using a rotating biological contactor. Biotechnology Progress, v. 19, p. 1372-1376, 2003.

ARNOLD, R. L. Dyes and Pigments. New Research, 1st ed, Nova Science Publishers, 2010.

BRASIL - Resolução CONAMA - Conselho Nacional do Meio Ambiente) No 430/2011 www.mma.gov.br/port/conama - acesso em 14/10/2013.

CHELLAMMAL, S.; KALAISELVI, P.; GANAPATHY, P.; SUBRAMANIAN, G. Anodic incineration of phthalic anhydride using $\mathrm{RuO}_{2}-\mathrm{IrO}_{2}-\mathrm{SnO}_{2}$. $-\mathrm{TiO}_{2}$ coated on $\mathrm{Ti}$ anode. Arabian Journal of Chemistry, 2012. http://dx.doi.org/10.1016/j. arabjc.2012.04.030.

COMNINELLIS, C. Electrocatalysis in the electrochemical conversion/combustion of organic pollutants for waste water treatment. Electrochimica Acta, v. 39, n. 11-12, p. 18571862, 1994.

DOS SANTOS, P. K. ; FERNANDES, K. C.; DE FARIA, L. A.; DE FREITAS, A. C.; DA SILVA, L. M. Descoloração e degradação do azo corante vermelho grlx-220 por ozonização. Química Nova, v. 34, n. 8, p. 1315-1322, 2011.

SONI, B. D.; PATEL, U. D.; RUPARELIA, J. P. Effects of $\mathrm{Ti} / \mathrm{RuO}_{2}-\mathrm{SnO}_{2}-\mathrm{Sb}_{2} \mathrm{O}_{5}$ electrode for decontamination of dyes from wastewater. Institute of Technology, Nirma University, Ahmedabad-382 481, 08-10 december 2011.

FORTI, J. C.; OLIVI P.; DE ANDRADE A. R. Ele- 
trochemical behavior of ethanol oxidation on a Ti/ $\mathrm{Ru}_{0,3} \mathrm{Ti}_{(0,7-x)} \mathrm{Sn}_{\mathrm{x}} \mathrm{O}_{2}$ eletrode: The role of experimental parameters in the selectivity of the products. Journal of the Eletrochemical Society, v. 150, n. 4, p. E222-E226, 2003.

FÓTI, G.; GANDINI, D.;COMNINELLIS, Ch. Anodic oxidation of organics on thermally prepared oxide electrodes. Current Topics in Eletrochemistry. v. 5, p. 71-91, 1997.

KARIYAJJANAVARA, P.; JOGTTAPPAA, N.; NAYAKAB, Y. A. Studies on degradation of reactive textile dyes solution by electrochemical method. Journal of Hazardous Materials, v. 190, p. 952-961, 2011.

KIM, I-H.; KIM, K-B. Ruthenium oxide thin film electrodes for super capacitors. Eletrochemical and Solid-State Letters, v. 2, p. 359-361, 1999.

KINOSHITA, E.; INGMAN, F.; EDWALL, G.; GLAB, S. An examination of the palladium-palladium oxide system and its utility for $\mathrm{pH}$ -sensing electrode. Eletrochimica Acta. v. 31, p. 29-38, 1986.

LANZA, M. R. V.; BERTAZZOLI, R. Selection of a commercial anode oxide coating for electro-oxidation of cyanide. Journal of the Brazilian Chemical Society, v. 13, n. 3, p. 345-351, 2002.

LASSALI, T. A. F.; BOODTS, J. F. C.; TRASATTI, $\mathrm{S}$. Electrocatalytic Activity of the ternary oxide $\mathrm{Ru}_{0,3} \mathrm{Pt}_{\mathrm{x}} \mathrm{Ti}_{(0,7-\mathrm{x})} \mathrm{O}_{2}$ for chlorine evolution. Electrochimica Acta, v. 39, n. 11-12, p. 1545-1549, 1994.

LIPP, L.; PLETCHER, D. The preparation and characterization of tin dioxide coated titanium electrodes. Electrochimica Acta, v. 42, n. 7, p. 1091-1099, 1997.

MARTÍNEZ-HUITLE, C. A.; BRILLAS, E. Decontamination of wastewaters containing synthetic organic dyes by electrochemical methods: A general review. Applied Catalysis B: Environmental, v. 87, p.105-145, 2009.

NEPPOLIAN, B.; CHOI. H. C.; SAKTHIVEL, S.; ARABINDOO, B.; MURUGESAN, V. Solar light induced and $\mathrm{TiO}_{2}$ assisted degradation of textile dye reactive blue 4 . Chemosphere v. 46 , p. 1173-1181, 2002.

NOGUEIRA, M. I. C. Tratamentos adsortivos e de oxidação avançada de fenol, 2-nitrofenol e 4-nitrofenol em solução aquosa. Tese (Doutorado) - Departamento de Engenharia Hidraúlica e Ambiental, Universidade Federal do Ceará, 175p, 2009.

NÚÑES, L.; GARCÍA-HORTAL, A.; TORRADES, F. Study of kinetic parameters related to the decolourization and mineralization of reactive dyes from textile dyeing using Fenton and photo-Fenton processes. Dye and Pigments, v.75, p. 647-652, 2007.

PAN, X.; USHIO, H.; OHSHIMA, T. Effects of molecular configurations of food colorants of their efficacies as photosensitizers in lipid oxidation. Food Chemistry, v. 92, p. 37- 44, 2005.

PANIZZA, M.; BOCCA, C.; CERISOLA, G. Electrochemical treatment of wastewater containing polyaromatic organic pollutants. Water Research, v. 34, n. 9, p. 2601-2605, 2000.

PANIZZA, M.; CERISOLA, G. Electrochemical processes for the treatment of organic pollutants. Advances in Chemistry Research, v. 2, p. 31-38, 2006.

PARSA, J. B.; GOLMIRZAEI, M; ABBASI, M. Degradation of azo dye C.I. Acid Red 18 in aqueous solution by ozone electrolysis process. Journal of Industrial and Engineering Chemistry (2013), http://dx.doi.org/10.1016/j. jiec.2013.05.034.

RAJESHWAR, K.; IBBANEZ, J. G. Environmental Eletrochemistry. Academic Press. London. 1996, 776p.

SOLIS M.; SOLIS A.; PEREZ H. I.; MANJARREZ, N.; FLORESA M. Microbial decolouration of azo dyes: A review. Process Biochemistry, v. 47, p. 1723-1748, 2012.

TEREZO, A. J.; PEREIRA, E. C. Preparation and characterization of $\mathrm{Ti} / \mathrm{RuO}_{2}$ anodes obtained by sol-gel and conventional routes. Materials Letters, v. 53, p. 339-345, 2002.

TORRES, R. A.; TORRES, W.; PERINGER, P.; PULGARIN, C. Electrochemical degradation p-substituted phenols of industrial interest on Pt electrodes. Attempt of a structure -reactivity relationship assessment. Chemosphere, v.50, p.97-104, 2003.

TRASATTI, S. Eletrochemistry and environ- 
ment: the role of eletrocatalysis. International Journal of Hyidrogen Energy, v.20, p.835-844, 1995.

WU Z.; JOO, H.; AHN, I. S.; HAAM, S.; KIM, J-H., LEE, K. Organic dye adsorption on mesoporous hybrid gels. Chemical Engineering Journal, v. 112 , p. $277-282,2004$.

\section{SOBRE OS AUTORES}

Maria Lucimar Maranhão Lima*

Engenheira Química pela Universidade Federal do Ceará. Mestre em Saneamento Ambiental pela Universidade Federal do Ceará. Doutoranda em Saneamento Ambiental pela Universidade Federal do Ceará/Pós-Graduação em Engenharia Civil/Saneamento Ambiental.

Ana Carine de Sousa Monte

Tecnóloga em Processos Químicos pelo Instituto Federal de Educação, Ciência e Tecnologia do Ceará.

Bárbara Costa Albuquerque

Tecnóloga em Processos Químicos pelo Instituto Federal de Educação, Ciência e Tecnologia do Ceará.

Maria Ionete Chaves Nogueira

Química Industrial pela Universidade Federal do Ceará. Mestre em Química Inorgânica pela Universidade Federal do Ceará. Doutora em Saneamento Ambiental pela Universidade Federal do Ceará.

Marisete Dantas de Aquino

Engenharia de Pesca pela Universidade Federal do Ceará. Mestre em Engenharia Civil pela Universidade Federal do Ceará, Doutora em Meio Ambiente e Recursos Hídricos pela École des Hautes Études en Sciences Sociales de Paris.

\section{Rinaldo dos Santos Araújo}

Químico Industrial pela Universidade Federal do Ceará. Mestre em Engenharia Química pela Universidade Federal do Rio Grande do Norte. Doutor em Química pela Universidade Federal do Ceará.

*Endereço: Universidade Federal do Ceará - Campus do Pici - Fortaleza-Ceará. Departamento de Engenharia Hidraúlica e Ambiental.

Tel.: 3366-9623.

E-mail: lucimar@ifce.edu.br 